\title{
Variación fenotípica del erizo Eucidaris tribuloides (Cidaroida: Cidaridae) en el Corredor Arrecifal del Suroeste del Golfo de México
}

\author{
Pastor Alberto Escarcega Quiroga ${ }^{1}$, Alejandro Granados Barba ${ }^{2 *}$, Carlos González Gándara ${ }^{3}$,
} Lucia Epherra ${ }^{4,5}$, Mark Marín-Hernández ${ }^{1} \&$ Augusto Crespi-Abril ${ }^{5,6}$

1. Maestría en Ecología y Pesquerías, Instituto de Ciencias Marinas y Pesquerías, Universidad Veracruzana, Boca del Río, Veracruz, México; escarcega.pastor@gmail.com

2. Instituto de Ciencias Marinas y Pesquerías, Universidad Veracruzana, Boca del Río, Veracruz, México; agranados1@gmail.com,markmarin@uv.mx

3. Laboratorio de Arrecifes Coralinos, Facultad de Ciencias Biológicas y Agropecuarias, Universidad Veracruzana, Tuxpan, Veracruz, México; cggandara@hotmail.com

4. Instituto de Diversidad y Evolución Austral (IDEAus-CONICET), Puerto Madryn, Chubut, Argentina; luepherra@gmail.com

5. Instituto Patagónico del Mar (IPaM)- Universidad Nacional de la Patagonia San Juan Bosco, Puerto Madryn, Chubut Argentina.

6. Centro para el Estudio de Sistemas Marinos (CESIMAR-CONICET), Puerto Madryn, Chubut, Argentina; augustocrespi@gmail.com

* Correspondencia.

$$
\text { Recibido 23-II-2019. Corregido 05-VI-2019. Aceptado 06-IX-2019. }
$$

\begin{abstract}
Phenotypic variation of sea urchin Eucidaris tribuloides (Cidaroida: Cidaridae) along the Reef Corridor of the Southwest Gulf of Mexico. Introduction: Intraspecific morphological variation can be attributed to the result of genetic variation or influence of environmental heterogeneity. In the latter case, organisms are exposed to diverse environmental conditions which have an influence on their biological processes and can be seen reflected in the morphological adaptations of species. Indeed, Reef Corridor in the Southwest Gulf of Mexico (CASGM, in its Spanish acronym) is constituted of geographically separated reefs that are exposed to different large-scale oceanographic factors and show their own attributes with multiple environmental variables. Therefore, this can stimulate morphological variations of species populations that are distributed in this corridor. Objective: The aim of this study was to determine the morphological variation of the Aristotle's lantern of the sea urchin Eucidaris tribuloides along CASGM. Methods: The allometric relation between height of the Aristotle's lantern and diameter of the test of 104 specimens was analyzed, and we also used a covariance analysis to detect allometric differences between groups. Apart from that, the variation of the shape of a rotule and a demi-pyramid for each sea urchin were analyzed using geometric morphometry. Results: There are allometric differences among reef systems in the north, center and south of Veracruz. The shape of rotula and demi-pyramid of sea urchins of the north zone are different from the central and south area. However, there were no differences in shape between the center and the South area. The centroid size of rotula and demi-pyramid of the sea urchins of the North are larger than those in the center and the South. Conclusions: Along the Corridor of the Southwest of the Gulf of Mexico, specimens of E. tribuloides showed a morphological variation in their analyzed structures, these results can be explained by the geographical and environmental gradients of the CASGM, in addition to the feeding habits of E. tribuloides and the availability of the food resource in the habitat where they are established. As a stimulus to the morphological variation found in this research, the distance among the reef systems and the marine currents patterns are also considered.
\end{abstract}

Key words: Aristotle's lantern; sea urchin; geometric morphometric; reefs; Gulf of Mexico.

Escarcega Quiroga, P. A., Granados Barba, A., González Gándara, C., Epherra, L., MarínHernández, M., \& Crespi-Abril, A. (2019). Variación fenotípica del erizo Eucidaris tribuloides (Cidaroida: Cidaridae) en el Corredor Arrecifal del Suroeste del Golfo de México. Revista de Biología Tropical, 67(6), 1146-1159. 
La variación morfológica intraespecífica puede ser el resultado del desarrollo ontogénico (Frédérich, Adriaens, \& Vandewalle, 2008; Díaz \& Campos, 2014), la variación genética (Frédérich, Liu, \& Dai, 2012) o la influencia de la heterogeneidad ambiental (Cardini, Jansson, \& Elton, 2007; Cardini \& Elton, 2009). En el último caso, los organismos están expuestos a diversas condiciones ambientales que influyen en sus procesos biológicos, lo cual, se puede reflejar en el comportamiento y las adaptaciones morfológicas de las especies (Cardini, Filho, Polly, \& Elton, 2010).

En el ambiente marino se presentan múltiples variables ambientales que podrían explicar la variación morfológica de las especies marinas a lo largo de gradientes ambientales y latitudinales (Márquez \& Van Der Molen, 2010; Nava-Ferrer \& Severeyn-Valbuena, 2011; Sánchez, Sepúlveda, Brante, \& Cárdenas, 2011). Particularmente, en los arrecifes de coral del océano Atlántico mexicano, se han reportado diferencias morfológicas intraespecíficas del pez Abudefduf saxatilis (Piñeros, Ríos-Cárdenas, Gutiérrez-Rodríguez, \& Mendoza-Cuenca, 2015) en respuesta a que los individuos se distribuyen en parches geográficamente separados, a lo largo de gradientes latitudinales, soportando diferentes condiciones ambientales (Arias-González, Legendre, \& RodríguezZaragoza, 2008).

El conjunto de arrecifes que se distribuyen a lo largo del litoral veracruzano denominado como el Corredor Arrecifal del Suroeste del Golfo de México (CASGM) por Ortiz-Lozano et al. (2013) tiene variables ambientales que cambian a lo largo del gradiente latitudinal, como es el caso de la salinidad, que disminuye en dirección norte-sur o la temperatura superficial del mar y la concentración de clorofila-a que aumentan en la misma dirección (Zavala-Hidalgo, Morey, \& O’Brien, 2003; SalasPérez, Ocaña-Valencia, \& González-Gándara, 2015). Por su lado, los patrones de corrientes marinas dentro del CASGM también cambian en el tiempo y el espacio (Salas-Monreal et al., 2017).
Se ha sugerido que dichas variaciones ambientales influyen en la composición, riqueza y distribución de algunas especies arrecifales. Tal es el caso de los equinodermos, que muestran variabilidad espacial de composición y riqueza entre el norte y sur de Veracruz, probablemente como resultado de la presión ambiental que ejerce la condición oligotrófica de las aguas en el norte y las eutróficas del sur (González-Gándara et al., 2015). Asimismo, se ha reportado que el ensamblaje coralino está estructurado en función de los gradientes de turbidez y productividad oceánica que hay a lo largo del CASGM (Jordán-Garza, GonzálezGándara, Salas-Pérez, \& Morales-Barragán, 2017). De este modo, el ambiente heterogéneo del corredor puede influir en la biología de los organismos que ahí se distribuyen. A pesar de ello, aún no se han desarrollado estudios para determinar el efecto geográfico y ambiental en la biología de las especies, específicamente en su morfología.

Se ha documentado que las características de algunas estructuras en erizos de mar tales como el grosor de la testa o caparazón, así como número y tamaño de las espinas se modifican por la intensidad de oleaje, la temperatura y por la presencia o ausencia de depredadores (Ebert, 1968; Dix, 1970; Marcus, 1980, 1983; Byrne \& Przeslawski, 2013; Byrne et al., 2014). Las estructuras funcionales de los erizos de mar como la linterna de Aristóteles (aparato masticador) también pueden presentar variación morfológica como respuesta al cambio en la disponibilidad del alimento ya que la fuerza de forrajeo aumenta cuando el recurso es limitado o cuando sus principales presas tienen estructuras calcáreas duras (Ebert, 1980; Hagen, 2008; Gianguzza \& Bonaviri, 2013; Epherra et al., 2015). De igual manera, la fuerza de forrajeo cambia dependiendo del tipo de sustrato en el que se encuentren. Si el sustrato es duro o plano, la linterna crecerá más respecto al diámetro de la testa, lo contrario ocurre si se desarrolla en sustratos blandos o con presencia de cavidades (Hernández \& Russell, 2009). También, las variaciones de salinidad generan cambios en el tamaño y peso de la linterna 
(Lau, Lau, Qian, \& Qiu, 2009), motivo por el cual puede ser una estructura que proporcione información sobre la plasticidad morfológica y al mismo tiempo sea un indicador de heterogeneidad ambiental.

Por lo anterior, los erizos de mar pueden ser modelos adecuados para estudiar la variación del tamaño y la forma como respuesta a las distintas presiones ambientales y gradientes latitudinales que se presentan en el litoral Veracruzano. Por ello, el presente estudio analiza la variación morfológica de la linterna de Aristóteles de Eucidaris tribuloides a lo largo del CASGM.

\section{MATERIALES Y MÉTODOS}

Área de estudio: El Corredor Arrecifal del Suroeste del Golfo de México (CASGM) cubre una distancia de $680 \mathrm{~km}$ lineales dentro de la plataforma continental del oeste del Golfo de México (Geissert-Kientz, 1999). Está conformado por sistemas de arrecifes coralinos de diferentes dimensiones que se distribuyen de norte a sur frente a la costa del estado de Veracruz, desde Tamiahua hasta Coatzacoalcos (Ortiz-Lozano et al., 2013). No obstante, se pueden distinguir tres grandes sistemas: el Sistema Arrecifal Lobos-Tuxpan (DOF, 2009), el Parque Nacional Sistema Arrecifal Veracruzano (DOF, 1992) y los Arrecifes de los Tuxtlas.

En el norte, el Sistema Arrecifal LobosTuxpan (SALT) se caracteriza por presentar una salinidad promedio de 36.5 ups (ZavalaHidalgo et al., 2003), una temperatura superficial promedio de $23.5^{\circ} \mathrm{C}$, variando de 20 a 30 ${ }^{\circ} \mathrm{C}$ y una concentración de clorofila-a de 0.76 $\mathrm{mg} / \mathrm{m}^{3}$, con mínimos de $0.2 \mathrm{mg} / \mathrm{m}^{3}$ y máximos de $1.6 \mathrm{mg} / \mathrm{m}^{3}$. Asimismo, está influenciado principalmente por el Río Tuxpan, cuyo promedio de descarga es de $138 \mathrm{~m}^{3} / \mathrm{s}$ (Salas-Pérez et al., 2015). Los sitios de recolección en el norte fueron los arrecifes; Blanquilla (2132'24.80'

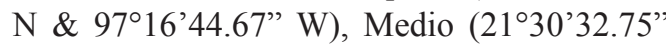

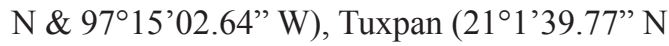
\& 97 $\left.91^{\circ} 38.45^{\prime \prime} \mathrm{W}\right)$ y Blake $\left(20^{\circ} 45^{\prime} 30^{\prime \prime} \mathrm{N} \&\right.$ $96^{\circ} 59^{\prime} 35^{\prime \prime} \mathrm{W}$ ).
En el centro, el Sistema Arrecifal Veracruzano (SAV) está definido por una salinidad promedio de 36 ups con un rango entre 34 y 36.5 ups, una temperatura superficial del mar de $24{ }^{\circ} \mathrm{C}$ en promedio, con un mínimo de $22^{\circ} \mathrm{C}$ y un máximo de $31^{\circ} \mathrm{C}$, mientras que la concentración de clorofila-a promedio es de $2.5 \mathrm{mg} / \mathrm{m}^{3}$ y oscila entre $0.9 \mathrm{mg} / \mathrm{m}^{3}$ y $4 \mathrm{mg} / \mathrm{m}^{3}$. Este sistema está influenciado principalmente por el río Jamapa, del cual se ha estimado una descarga promedio de $45 \mathrm{~m}^{3} / \mathrm{s}$ con máximos de $380 \mathrm{~m}^{3} / \mathrm{s}$ y mínimos de $8 \mathrm{~m}^{3} / \mathrm{s}$ (Perales-Valdivia, Sanay-González, \& Marín-Hernández, 2015; Salas-Pérez et al., 2015) y también hay influencias de los ríos La Antigua y Papaloapan (Krutak, 1997). Los sitios de recolección fueron los arrecifes; La Segunda Laja (19 $28^{\prime} 44^{\prime \prime}$ $\mathrm{N} \& 96^{\circ} 18^{\prime} 24^{\prime \prime} \mathrm{W}$ ) en las inmediaciones de la localidad de Chachalacas y en la Anegada de adentro (19¹3'54.8” $\mathrm{N} \&$ 96³'27.5” W).

En el sur, los Arrecifes de los Tuxtlas (AT) están caracterizados por presentar salinidad promedio de 35.5 ups (Zavala-Hidalgo et al., 2003), temperaturas superficiales promedio de $25.64{ }^{\circ} \mathrm{C}$ con mínimos de 22 y $30.5{ }^{\circ} \mathrm{C}$, una concentración de clorofila-a promedio de 4.9 $\mathrm{mg} / \mathrm{m}^{3}$ y un rango de 0.82 y $10.91 \mathrm{mg} / \mathrm{m}^{3}$. Este sistema está influenciado principalmente por el río Coatzacoalcos con una descarga promedio de $353 \mathrm{~m}^{3} / \mathrm{s}$ (Salas-Pérez et al., 2015). Los sitios de recolección fueron los arrecifes; Zapotitlán (18³0'56.9” N \& 9447'12.8” W), Balzapote $\left.18^{\circ} 37^{\prime} 20^{\prime \prime} \mathrm{N} \& 95^{\circ} 3^{\prime} 58.3^{\prime \prime} \mathrm{W}\right)$, La Perla (18³2’33.8' N \& 9449'4.1”'W), Tripié (18 $10^{\prime} 33.6 ”$ N \& 94²2’03”'W) y Palo Seco (18¹0’33.1” N \& 94³1'32” W).

Las corrientes superficiales varían en el tiempo y el espacio dentro del corredor. La dirección de las corrientes costeras de Veracruz es de norte-sur de septiembre a marzo, mientras que de abril a agosto la dirección es de sur-norte (Zavala-Hidalgo et al., 2003). Por otro lado, la información sobre las corrientes superficiales del mar Caribe y Golfo de México proporcionadas por la Administración Nacional de la Atmósfera y el Océano, 2015 (NOAA) a través del Nodo Regional del Caribe y Golfo de México Coast-Watch (http://cwcaribbean.aoml. 
noaa.gov/data.html) evidencian giros ciclóni$\cos$ y anticiclónicos temporales frente a las costas de Veracruz, lo cual, puede influir en la dispersión larval de las especies a lo largo del CASGM (Salas-Monreal et al., 2018).

Manejo de ejemplares: Se tomaron 26 ejemplares de Eucidaris tribuloides de la Colección de Invertebrados de la Universidad Veracruzana (número de catálogo: DF-CC279-13; muestras tomadas de marzo a octubre 2013). Además, se recolectaron 77 individuos en campo para completar el tamaño de la muestra previamente establecida (marzo a junio 2016). Considerando la máxima talla de madurez sexual registrada por McPherson (1968), se procuró capturar ejemplares mayores de 25 $\mathrm{mm}$ de diámetro de testa para evitar variación relacionada con la madurez sexual. En total, se analizaron 104 individuos de los cuales 30 corresponden al SALT (17 en campo y 13 de colección), 35 al SAV y 39 (26 en campo y 13 de colección) al SAT.

Una vez capturados los ejemplares, se narcotizaron dejándolos reposar en un recipiente oscuro con suficiente agua marina y una cucharada de cloruro de magnesio durante $1 \mathrm{~h}$. Luego se fijaron en alcohol etílico al $96 \%$ durante 24 $\mathrm{h}$, se etiquetaron con los siguientes datos: recolector, localidad, profundidad, fecha de colecta y posición geográfica del sitio de recolecta y finalmente se conservaron en seco. Las espinas de cada erizo de mar se removieron con una inmersión de hipoclorito de sodio durante $10 \mathrm{a}$ $20 \mathrm{~min}$, esto para obtener las medidas lineales más precisas del diámetro de la testa. Se extrajo la linterna de Aristóteles de cada individuo y se registró la altura de la estructura, todas las medidas se obtuvieron con un vernier digital con precisión de $0.01 \mathrm{~mm}$. Posteriormente, se removió el tejido adherido a la linterna de Aristóteles con una inmersión en hipoclorito de sodio al $10 \%$ durante $24 \mathrm{~h}$ para desarticular los osículos de la linterna.

Análisis estadístico de morfometría clásica: Con el diámetro de la testa y la altura de la linterna se realizó una regresión para cada grupo (norte, centro y sur) con el fin de calcular la alometría entre el diámetro de la testa versus la altura de la linterna. Un análisis de covarianza (ANCOVA) fue utilizado para comparar las pendientes de esas regresiones a un nivel de significancia $\alpha<0.05$, donde la altura de la linterna de Aristóteles fue utilizada como variable dependiente, las zonas; norte, centro y sur como variables agrupadas y el diámetro de la testa como covariable. Lo anterior se realizó probando previamente la homogeneidad de las pendientes y con el análisis de Levene se probó homogeneidad de varianzas de los datos mediante el Software estadístico SPSS.

Morfometría geométrica: Se seleccionó una rótula por cada linterna de Aristóteles para obtener imágenes fotográficas de su parte aboral (Fig. 1A). Las imágenes se tomaron en el Laboratorio de Microscopía y Fotografía de la Biodiversidad en el Instituto de Biología de la UNAM utilizando una cámara LEICADFC490 de 8 megapíxeles que viene integrada a un microscopio estereoscópico multifocal LEICA-Z16APOA, manipulado desde un ordenador mediante el software "Leica Application Suite" (LAS por sus siglas en inglés). Asimismo, se obtuvieron imágenes fotográficas de una semipirámide por cada linterna orientado en su plano lateral interno con una cámara de 16 megapíxeles de la marca Panasonic DMHZS10 (Fig. 1C).

Posteriormente, se colocaron landmarks y semilandmarks con el software TpsDig 2.26 (Rohlf, 2016). Estos son puntos homólogos que se colocan sobre las imágenes de las estructuras a estudiar considerando los criterios de Bookstein (1991). En este estudio, se asumió simetría bilateral para el caso de la rótula. Por lo tanto, se analizó el lado derecho de cada estructura para evitar redundancia de información. La configuración consistió de seis landmarks y seis semilandmarks para caracterizar la forma del lado derecho de la rótula (Fig. 1B). El criterio que se utilizó para colocar los semilandmarks consistió en poner seis líneas equidistantes entre sí y transversales al contorno de la rótula con el software Makefan8 (IMP) 

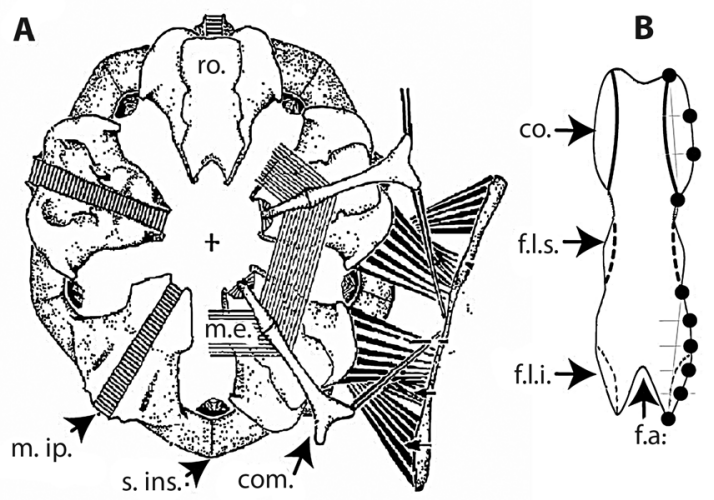

C

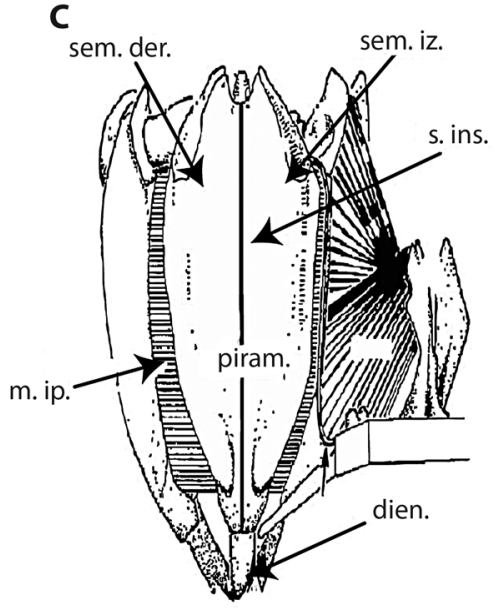

Abreviaturas

com. Compás

co. Cóndilo

ro. Rótula

f.a. Fosa anterior

f.l.i. Fosa lateral inferior

f.l.s. Fosa lateral superior

m.e. Músculo elevador

m.ip. Músculo interpiramidal

s.ins. Sutura intersemipiramidal

Fig. 1. (A) Linterna de Aristóteles vista en el plano superior, (B) Rótula parte aboral y sus landmarks y semilandmarks, (C) Linterna de Aristóteles vista desde el plano vertical, (D) Semipirámide vista lateral interno y sus landmarks. Imagen modificada de Märkel (1979).

Fig. 1. (A) Aristotle's lantern view from above, (B) Rotula view from above and its landmarks and semilandmarks, (C) Aristotle's lantern view from the side, (D) Left demipyramid inner side and its landmarks. From Märkel (1979).

(Sheets, 2014a), posterior a ello, se colocaron los semilandmarks en el punto de intercepción entre las líneas y el contorno (Fig. 1B). Para el caso de la semipirámide se establecieron seis landmarks figura (Fig. 1D).

Se realizó un ajuste de Procrustes a la configuración de coordenadas de cada estructura analizada, método de superposición que consiste en eliminar todos los efectos por tamaño, posición y rotación de los objetos, descartando así la variación de la forma no biológica. Para el caso particular de la rótula, los semilandmarks se ajustaron con el método de "minimum bending energy" usando el Software TpsRelw
1.65 (Rholf, 2016). También, se extrajo el valor del tamaño centroide (información completa del tamaño) de cada estructura y se aplicó la función de logaritmo natural para normalizar los datos. Con ello, se realizaron ANOVAs para comparar el tamaño centroide de las rótulas y semipirámides entre los grupos norte, centro y sur del CASGM. Consecutivamente, se aplicó una prueba de Tukey para comparar las medias de las muestras en caso de presentar diferencias significativas $(\alpha<0.05)$.

Se efectuó una regresión multivariada de las coordenadas Procrustes (variable dependiente) sobre el tamaño centroide de la rótula y 
semipirámide (variable independiente), con la finalidad de observar si el cambio de la forma de la rótula y semipirámide se debía al tamaño. También se realizó un Análisis de Componentes Principales Entre Grupos (BGPCA por sus siglas en inglés) utilizando la configuración promedio de las coordenadas Procrustes de cada grupo (norte, centro y sur), esto con base en las sugerencias de Mitteroecker y Bookstein (2011). Además, se utilizaron gradillas de deformación para observar los patrones e intensidad de la variación morfológica a lo largo de los ejes del BGPCA en el software PcaGeen8 (IMP) (Sheets, 2014b), Dichas gradillas permiten visualizar con mejor precisión la ubicación anatómica con mayor variación morfológica de las estructuras analizadas. Finalmente, se extrajo los "partial warp scores" o variables de la forma con ayuda del Software CoordGen8 (Sheets, 2014c) de la serie IMP8 para realizar un Análisis Multivariado de la Varianza (MANOVA por sus siglas en ingles) para determinar si existen diferencias morfológicas entre los individuos del norte, centro y sur del CASGM.

\section{RESULTADOS}

Morfometría clásica: El diámetro de la testa de E. tribuloides osciló entre 20 y 45 $\mathrm{mm}$, con un promedio de $30.44 \mathrm{~mm}$ y una desviación estándar de $4.89 \mathrm{~mm}$, mientras que la altura de la linterna de Aristóteles osciló entre 9 y $18 \mathrm{~mm}$ con un promedio de $14.5 \mathrm{~mm}$ y una desviación estándar de $2.42 \mathrm{~mm}$. La relación entre esas dos estructuras fue lineal en todos los sitios. Los individuos del norte presentaron las linternas más grandes, mientras que los del sur las más pequeñas (Fig. 2). La prueba de Levene no mostró diferencias significativas entre grupos $(\mathrm{F}=2.18, \mathrm{P}=0.119)$ y la interacción entre las zonas y la altura de la linterna indicó que las pendientes fueron homogéneas $(\mathrm{F}=0.964$, $\mathrm{P}=0.386$ ). Por su parte, el ANCOVA manifestó diferencias significativas entre grupos $(\mathrm{F}=10.74, \mathrm{P}<0.001)$ con un coeficiente de regresión de $86 \%$, mientras que la comparación por pares con ajuste de Bonferroni señaló que el norte es diferente al centro $(\mathrm{SE}=0.249$, $\mathrm{P}=0.002)$ y el sur $(\mathrm{SE}=0.253, \mathrm{P}<0.001)$. Sin embargo, no hubo diferencias entre centro y sur $(\mathrm{SE}=0.226, \mathrm{P}=0.771)$.

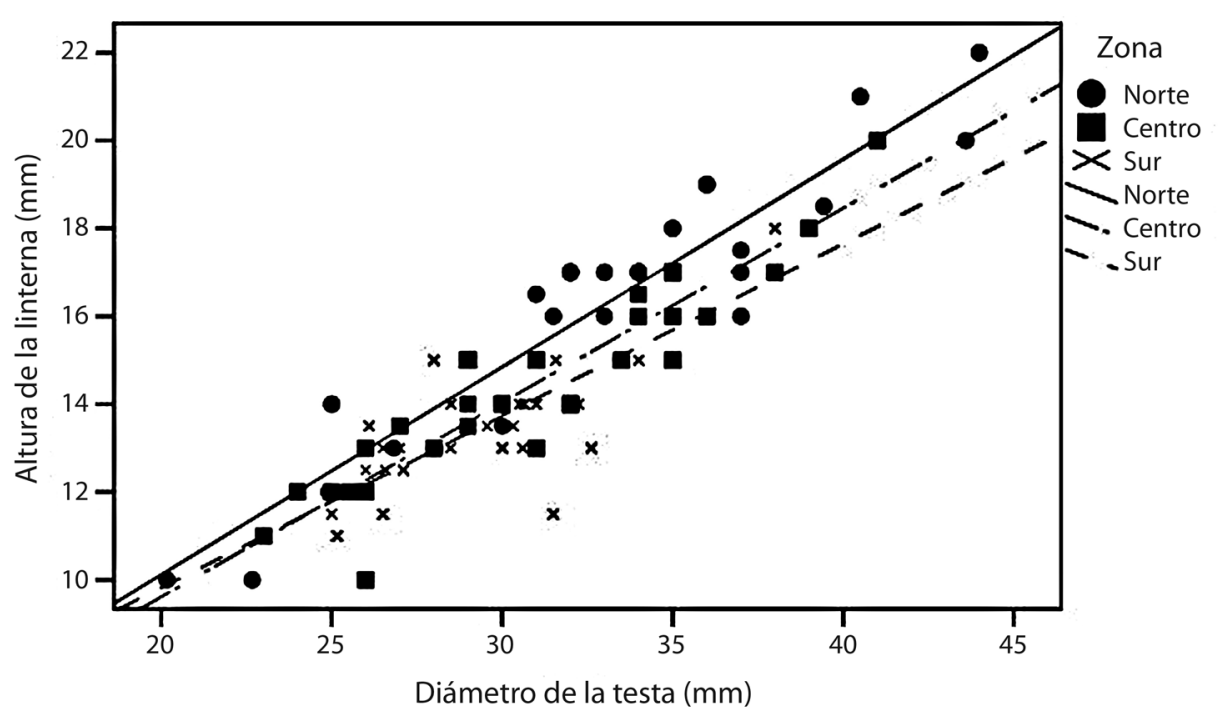

Fig. 2. Relación del diámetro de la testa versus altura de la linterna de Aristóteles de Eucidaris tribuloides para cada sitio estudiado (norte, centro y sur de Corredor Arrecifal del Suroeste del Golfo de México).

Fig. 2. Allometric relationship between test diameter and Aristotle's lantern of Eucidaris tribuloides length to each site (north, center and south from Corridor of the Southwestern Gulf of Mexico). 
Morfometría geométrica: El análisis de varianza (ANOVA) confirmó la existencia de diferencias significativas entre los grupos norte, centro y sur para el logaritmo natural del tamaño centroide de la rótula $(\mathrm{F}=8.02 ; \mathrm{P}$ $=0.001)$ y semipirámide $(\mathrm{F}=5.96 ; \mathrm{P}=0.004)$. La prueba post hoc de Tukey indica que el tamaño de la rótula de los erizos del norte es significativamente mayor que los del centro y sur. Por otro lado, el tamaño de la semipirámide de los organismos del norte es diferente a la de los individuos del sur. Sin embargo, no hubo diferencias significativas entre individuos del centro y norte, tampoco entre los individuos del centro y el sur.

El análisis de regresión múltiple explicó el $4.6 \%$ de la variación de la forma para la rótula y el $11.26 \%$ para semipirámide, lo que sugiere una posible ausencia de correlación entre el tamaño y la forma de la linterna de Aristóteles para Eucidaris tribuloides. Los MANOVAs confirmaron la existencia de diferencias significativas entre los grupos norte, centro y sur para la rótula (Lambda de Wilks $=0.384, \mathrm{P}=$ 0.001) y semipirámide (Lambda de Wilks = $0.6432, \mathrm{P}<0.0001)$. La comparación por pares mediante la $\mathrm{T}^{2}$ de Hotelling mostró que el norte fue significativamente diferente al sur para la rótula y semipirámide (Tabla 1).

Por otro lado, el Análisis de Componentes Principales Entre Grupos para la rótula mostró la distribución de los individuos en dos ejes, el primero contiene $75.36 \%$ de la variación de la forma. La proyección de los individuos sobre los dos ejes canónicos reveló solapamiento entre los sistemas arrecifales. Pese a ello, se observa que los individuos del norte presentan mayor variación morfológica en comparación con los individuos del sur (Fig. 3A). Para el caso de la semipirámide, la distribución de los individuos fue sobre dos ejes canónicos de los cuales el primer eje contuvo el $87.38 \%$ de la variación. La proyección de los individuos sobre los dos ejes canónicos presenta gran solapamiento entre los grupos del norte, centro y sur. Sin embargo, se observa que los individuos

TABLA 1

Distancia de Mahalanobis entre sistemas arrecifales y valor de significancia de la prueba post hoc mediante $\mathrm{T}^{2}$ de Hotelling

TABLE 1

Mahalanobis distance between reef systems and significance value of the post hoc test by Hotelling's T ${ }^{2}$

\begin{tabular}{cccccc}
\multicolumn{2}{c}{ Zonas } & \multicolumn{2}{c}{ Rótula } & \multicolumn{2}{c}{ Semipirámide } \\
\cline { 3 - 6 } norte & Centro & 5.94 & 0.003 & 1.42 & 0.142 \\
norte & Sur & 4.99 & 0.009 & 1.9 & 0.009 \\
centro & Sur & 1.49 & 1.495 & 1.05 & 0.248 \\
\hline
\end{tabular}

D.M.= Distancia de Mahalanobis, $\mathrm{P}=$ valor de significancia .

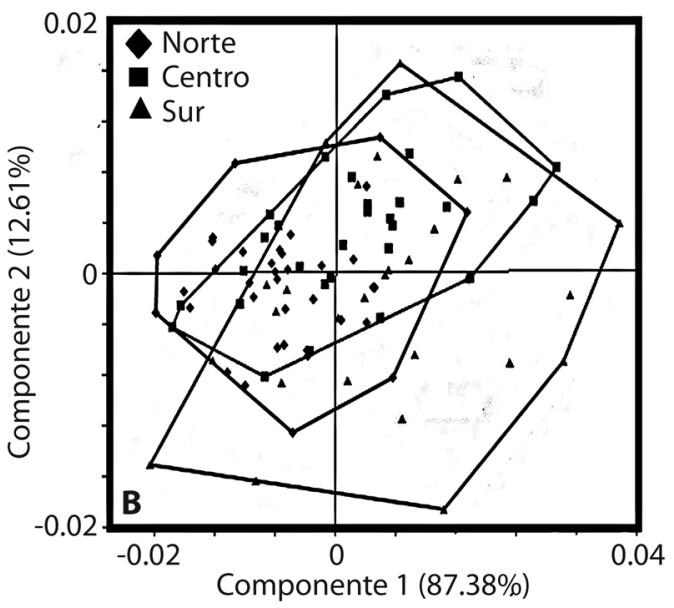

Fig. 3. Análisis de Componentes Principales entre grupos de la rótula (A) y Semipirámide (B) de Eucidaris tribuloides. Fig. 3. Between-Groups Principal Component Analyses of rotula (A) and demi-pyramid (B) of Eucidaris tribuloides. 
del norte presentaron mayor variación que los individuos del sur (Fig. 3B).

Las rejillas de deformación revelaron algunas diferencias en la forma de la rótula, principalmente en el cóndilo, que es más robusto en los individuos del centro. También, la fosa lateral superior de los individuos del norte es angosta (Fig. 4). Para el caso de la semipirámide, se revelaron algunas diferencias morfológicas donde los individuos que se distribuyeron sobre los valores negativos de la primera función discriminante de la figura $3 \mathrm{~B}$ son más robustas. Por el contrario, los individuos que se
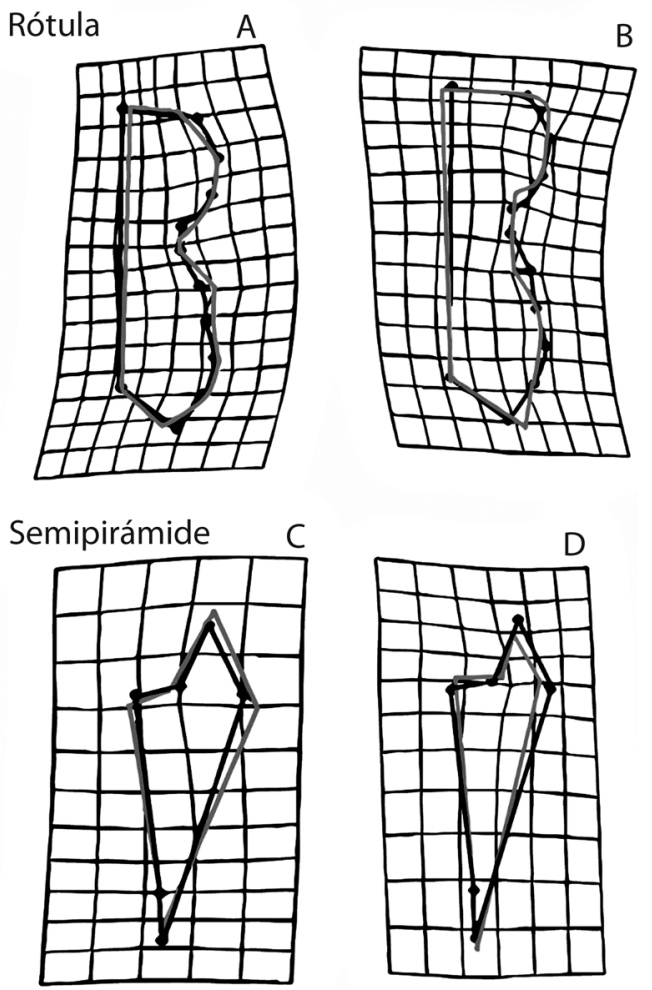

Fig. 4. Variación de la forma de la rótula y semipirámide a lo largo de la primera función discriminante de la figura 3 , cada rejilla mostró la predicción de la forma para A) -0.1 y B) 0.1 , las figuras de color negro muestran la forma promedio y las figuras de color gris muestran la variación de la forma.

Fig. 4. Variation of the shape from rotula and semipyramid along the first discriminant function of the figure 3, B. Each grid showed the prediction of shape to A) -0.1 and B) 0.1 , the black figures show the average shape and the gray figures show the variation of the shape. distribuyeron sobre los valores positivos de la primera función discriminante de la figura $3 \mathrm{~B}$ poseen semipirámides más estrechas. Por otro lado, existe una notoria variación de la forma en la parte superior de la semipirámide (Fig. 4).

\section{DISCUSIÓN}

La morfometría geométrica es una herramienta que sirve para detectar cambios sutiles de la forma (Zelditch, Swiderski, \& Sheets, 2012). En biología, estos cambios pueden ser atribuidos a los procesos de especiación (Binks, Evans, Kennington, \& Price, 2011), a la baja o nula interacción genética entre poblaciones (Frédérich et al., 2012) o a la plasticidad morfológica (Vega-Sánchez, Camacho-Morales, Chassin-Nora, \& Mendoza-Cuenca, 2012). Debido a esto, los estudios de morfometría geométrica a lo largo de gradientes latitudinales o ambientales han servido en la práctica para determinar stocks pesqueros (Cadrin \& Friedland. 1999; Márquez, Robledo, Peñaloza, \& Van der Molen., 2009) o establecer estructuras espaciales finas de poblaciones locales o regionales (Schachter-broide, Dujardin, Kitron, \& Gürtler, 2004). En el presente estudio, fue una herramienta que, aplicada a la linterna de Aristóteles de Eucidaris tribuloides, sirvió como indicador de heterogeneidad ambiental.

La relación entre la longitud de la linterna de Aristóteles y el diámetro de la testa de E. tribuloides fue diferente entre los grupos analizados (norte, centro y sur del CASGM), probablemente como resultado del desarrollo ontogénico, o bien por la influencia de los factores genéticos o ambientales que pueden limitar o estimular el crecimiento de alguna de las estructuras analizadas, influyendo así en la variación alométrica entre grupos poblacionales (Klingenberg, 2016). Precisamente, para el caso de los erizos de mar se ha demostrado que la presión ambiental puede influir en el crecimiento de la testa y la linterna de Aristóteles de los erizos de mar (Lawrence \& Jangoux, 2013).

Mediante la morfometría geométrica se observó la ausencia de una relación alométrica entre el tamaño centroide y la forma de la 
rótula y semipirámide de la linterna de Aristóteles de E. tribuloides. Esto sugiere que el tamaño de las estructuras analizadas no afectó la variación morfológica observada en esta investigación. Asimismo, Epherra et al. (2015) obtuvieron resultados similares para el caso de la semipirámide de Arbacia dufresnii. Sin embargo, ocurrió lo contrario para la rótula de dicha especie. Esto puede ser debido a las diferencias en los diseños de muestreo, ya que en $A$. dufresnii se analizaron individuos con diámetros de testa que van de 10 a $50 \mathrm{~mm}$, mientras que en el presente estudio se recolectaron individuos mayores a $20 \mathrm{~mm}$ de diámetro de testa para evitar la variación morfológica debido al estado de madurez sexual. Con base en lo anterior, es posible que la variación morfológica en erizos de mar también se manifieste por efecto del desarrollo ontogénico (Frédérich et al., 2008; Díaz \& Campos, 2014).

Por otro lado, la variación morfológica de la linterna de Aristóteles para erizos herbívoros está influenciada por la disponibilidad de algas, es decir, cuando la cobertura o disponibilidad de algas es baja, la fuerza de forrajeo aumenta. Por lo tanto, la linterna de Aristóteles crece más en relación al diámetro de la testa (Ebert 1980; Black, Johnson, \& Trendall, 1982; Levitan, 1988). Esto podría explicar la variación morfológica observada en el presente estudio, ya que, posiblemente la disponibilidad de alimento representado por la cobertura de algas difiere entre los sitios estudiados, lo que estimularía la variación morfológica de la linterna de Aristóteles E. tribuloides.

Sin embargo, E. tribuloides es una especie omnívora (Lawrence \& Jangoux, 2013) y por ello, puede alimentarse de organismos blandos como: tapetes de algas, pastos marinos, esponjas y organismos intersticiales de sedimentos limosos, así como de organismos con estructuras duras, tales como: algas calcáreas, foraminíferos, corales, gasterópodos y bivalvos (McPherson, 1968; Kier, 1975; Lawrence \& Glynn, 1984; Cutress, 1965; Santos, Coutinho, \& Hajdu, 2002). Dicho comportamiento pudo estimular el crecimiento de la linterna de Aristóteles en erizos que se distribuyeron sobre sustratos duros o compuestos principalmente por organismos con estructuras duras y limitar el crecimiento de aquellas linternas de erizos que se encontraron sobre sustrato blando o compuesto principalmente por organismos blandos (Hagen, 2008; Gianguzza \& Bonaviri, 2013).

Por otra parte, el CASGM tiene un gradiente de salinidad que disminuye con dirección norte-sur, así como gradientes de temperatura superficial de mar y concentración de clorofilacreciente en la misma dirección (Zavala-Hidalgo et al., 2003: Salas-Pérez et al., 2015). Lo anterior puede estar relacionado con la longitud relativa de la linterna de Aristóteles y el tamaño centroide de la rótula y semipirámide de $E$. tribuloides que gradualmente disminuyen hacia el sur. Esto es posible, por un lado, debido las fluctuaciones de salinidad que pueden limitar el crecimiento de la testa y semipirámide de algunos erizos de mar cuando la salinidad es baja (Lau et al., 2009). Por otro lado, la temperatura superficial del mar y la concentración de clorofila-a también pueden estar asociados a los cambios morfológicos de la linterna de Aristóteles de E. tribuloides. Sin embargo, no hay suficiente información que demuestre el efecto de las fluctuaciones de temperatura y concentración de clorofila-a sobre la morfología y crecimiento de la linterna de Aristóteles de los erizos de mar. No obstante, estos factores junto con los atributos propios de cada arrecife tales como el tipo de sustrato, profundidad, complejidad y la distancia a la costa, representan un papel importante en la definición del sustrato y la estructura comunitaria de los organismos bentónicos de cada arrecife (Arias-González et al., 2006, 2008; Jordán-Garza et al., 2017). Esto a su vez puede influir en la dieta de $E$. tribuloides y consecutivamente podría tener un efecto indirecto en la variación morfología de la linterna de Aristóteles debido a la capacidad de adaptación alimenticia que presenta esta especie (Lawrence \& Jangoux, 2013).

Dicho lo anterior, es importante considerar que algunas muestras se capturaron en el año 2013 y otras en el año 2016. Ello, podría influir en los resultados de esta investigación, 
dado que las comunidades bentónicas de los arrecifes coralinos son complejos y dinámicos, por lo que la estructura de las comunidades bentónicas de los arrecifes puede variar a lo largo de un corto periodo de tiempo (Ceccarelli, Richars, Pratchett, \& Cvitanovic, 2011). Esto, también podría influir en la dieta y por lo tanto en la variación morfología de la linterna de Aristóteles de E. tribuloides. Sin embargo, los resultados de esta investigación muestran diferencias entre los grupos del norte y sur, sitios que tienen proporción similar de ejemplares de colección capturados en 2013. Además, no se encontraron diferencias entre el sur y el centro, en este último, todos los ejemplares se capturaron en el año 2016. Por lo tanto, se infiere que este factor no es relevante en los resultados encontrados.

Por otro lado, la disminución del flujo genético debido a la distancia geográfica entre localidades puede dar lugar a la divergencia fenotípica (Slatkin, 1987; Hendry, Day, \& Taylor, 2001) detectada en la morfología de la rótula y la semipirámide de los erizos del norte (SALT) versus centro (SAV) y sur (SAT). Por otra parte, el patrón de las corrientes marinas en el corredor arrecifal veracruzano (SalasMonreal et al., 2018) conecta a las poblaciones de erizos pero en algunos casos pueden actuar como barreras para el intercambio genético entre poblaciones geográficamente separadas (Cowen, Gawarkiewicz, Pineda, Thorrold, \& Werner, 2007), especialmente cuando hay barreras locales, tal como se ha reportado para caso del pez Stegastes partitus que mostró un limitado intercambio genético entre subpoblaciones de los sistemas arrecifales del SAV (VillegasSánchez, Pérez-España, Rivera-Madrid, SalasMonreal, \& Arias-González, 2014).

En contraste, las subpoblaciones del pez Abudefduf saxatilis que se distribuyen en el norte y centro de Veracruz presentan homogeneidad genética. Ello, a pesar de las diferencias morfológicas detectadas entre dichas subpoblaciones lo cual, indica que las poblaciones de esta especie están interconectadas genéticamente entre los arrecifes veracruzanos, pero el ambiente ejerce presión que afecta su morfología (Piñeros et al., 2015). Esto mismo puede estar ocurriendo con E. tribuloides, debido a que se reproduce sexualmente por fecundación externa, presenta larvas planctónicas y son sedentarios en su etapa adulta (Hendler, Miller, Pawson, \& Kier, 1995; Lawarence \& Jangoux, 2013). Dicho estilo de vida podría permitir que esta especie mantenga un buen intercambio genético entre subpoblaciones y se disperse a lo largo del CASGM, además de soportar la presión ambiental local durante su etapa juvenil y adulta, sugiriendo que el origen de la variación morfológica detectada en esta investigación se debe a la presión ambiental local y no a la baja interacción genética entre los grupos analizados.

En esta investigación, la mayoría de los individuos que representaron al centro (con 31 ejemplares recolectados) provienen del arrecife La Segunda Laja, el cual se encuentra $10 \mathrm{~km}$ al norte del SAV. Pese a ello, dicho arrecife tiene características similares a los arrecifes del sur, ya que es de tipo marginal, somero, con baja cobertura coralina y un sustrato compuesto principalmente por sedimento y tapetes de algas (observaciones personales). Lo anterior también puede explicar la ausencia de diferencias morfológicas entre los individuos del centro y el sur del CASGM.

En conclusión, los individuos de E. tribuloides a lo largo del Corredor Arrecifal del Suroeste del Golfo de México mostraron variación morfológica en sus estructuras analizadas. Estas diferencias morfológicas pueden estar relacionadas al diseño de muestreo, factores genéticos o bien a las múltiples variables ambientales, incluyendo la salinidad, temperatura, concentración de clorofila-a y las variables propias de cada arrecife, tales como la cobertura bentónica y el tipo de sustrato en el que se encuentran. Nosotros sugerimos que estas variables ambientales pueden tener un efecto directo o indirecto sobre la variación morfología de la linterna de Aristóteles de $E$. tribuloides al adaptar sus hábitos alimenticios y ajustar el rendimiento de ramoneo de acuerdo a la disponibilidad del alimento y las características del entorno particular de cada arrecife. 
Finalmente, es necesario estudiar con mayor profundidad el tema del efecto geográfico, genético y ambiental sobre la variación morfológica de los organismos en CASGM, con la finalidad de tener información más precisa sobre las causas que estimulan la variación morfológica en el corredor, esto puede ser útil para identificar heterogeneidad ambiental, proponer unidades o subunidades de manejo, y (en caso de requerirse) establecer stocks pesqueros, información que es necesaria para el manejo y administración de los recursos del Corredor Arrecifal Suroeste del Golfo de México.

Declaración de ética: los autores declaran que todos están de acuerdo con esta publicación y que han hecho aportes que justifican su autoría; que no hay conflicto de interés de ningún tipo; y que han cumplido con todos los requisitos y procedimientos éticos y legales pertinentes. Todas las fuentes de financiamiento se detallan plena y claramente en la sección de agradecimientos. El respectivo documento legal firmado se encuentra en los archivos de la revista.

\section{RESUMEN}

Introducción: La variación morfológica intraespecífica puede ser el resultado del desarrollo ontogénico, la variación genética o la heterogeneidad ambiental. En el último caso, los organismos están expuestos a diversas condiciones ambientales, lo que puede influir en el comportamiento y las adaptaciones morfológicas de las especies. Precisamente, el Corredor Arrecifal del Suroeste del Golfo de México (CASGM) está compuesto por arrecifes separados geográficamente que están expuestos a diferentes factores oceanográficos, así como atributos propios con múltiples variables ambientales. Por lo tanto, es posible encontrar diferencias morfológicas de las poblaciones de especies que se distribuyen en el corredor. Objetivo: Determinar la variación morfológica de la linterna de Aristóteles del erizo de mar Eucidaris tribuloides a lo largo del CASGM. Métodos: Se analizó la relación alométrica entre la altura de la linterna versus diámetro de la testa en 104 especímenes, también realizamos un análisis de covarianza para detectar diferencias alométricas entre grupos. Se analizó la variación de la forma de una rótula y una semipirámide de cada erizo de mar mediante morfometría geométrica. Resultados: Existen diferencias alométricas entre sistemas arrecifales. La forma de la rótula y semipirámide de los erizos de mar del norte son diferentes a los erizos del centro y el sur; sin embargo, no hubo diferencias de forma entre el centro y el sur. El tamaño centroide de la rótula y semipirámide de los erizos de mar del norte son significativamente más grandes que los del centro y el sur. Conclusiones: A lo largo del Corredor Arrecifal del Suroeste del Golfo de México, los individuos de E. tribuloides mostraron variación morfológica en sus estructuras analizadas, dichos resultados, pueden ser explicados por los gradientes latitudinales y ambientales del CASGM, además de los hábitos alimenticios de la especie y la disponibilidad del recurso alimenticio en el ambiente donde se establecen.

Palabras clave: morfometría geométrica; linterna de Aristóteles; erizo de mar; arrecifes; Golfo de México.

\section{REFERENCIAS}

Arias-González, J. E., Done, T. J., Page, C. A., Cheal, A. J., Kininmonth, S., \& Garza-Pérez, J. R. (2006). Towards a reef scape ecology: relating biomass and trophic structure of fish assemblages to habitat at Davies Reef, Australia. Marine Ecology Progress Series, 320, 29-41.

Arias-González, J. E., Legendre, P., \& Rodríguez-Zaragoza, F. A. (2008). Scaling up beta diversity on Caribbean coral reefs. Journal of Experimental Marine Biology and Ecology, 366, 28-36.

Binks, R. M., Evans, J. P., Kennington, W. J., \& Price, J. (2011). Spatial patterns of variation in color and spine shape in the sea urchin Heliocidaris erythrogramma. Invertebrate Biology, 130, 161-174.

Black, R., Johnson, M. S., \& Trendall, J. T. (1982). Relative size of Aristotle's lantern in Echinometra mathaei occurring at different densities. Marine Biology, 71, 101-106.

Bookstein, F. L. (1991). Morphometric tools for landmark data: Geometry and Biology. Cambridge, United Kingdom: Cambridge University Press.

Byrne, M., \& Przeslawski, R. (2013). Multistressor impacts of warming and acidification of the ocean on marine invertebrates' life histories. Integrative and Comparative Biology, 53, 582-596.

Byrne, M., Smith, A. M., West, S., Collard, M., Dubois, P., Graba-landry, A., \& Dworjanyn, S. A. (2014). Warming influences $\mathrm{Mg}^{2+}$ content, while warming and acidification influence calcification and test strength of a sea urchin. Environmental Science \& Technology, $48,12620-12627$.

Cadrin, S. X., \& Friedland, K. D. (1999). The utility of image processing techniques for morphometric analysis and stock identification. Fisheries Research, 43, 129-139. 
Cardini, A., \& Elton, S. (2009). Geographic and taxonomic influences on cranial variation in red colobus monkeys (Primates, Colobinae): introducing a new approach to 'morph' monkeys. Global Ecology and Biogeography, 18(2), 248-263.

Cardini, A., Filho, J. A. F. D., Polly, P. D., \& Elton, S. (2010). Biogeographic analysis using geometric morphometrics: clines in skull size and shape in a widespread African arboreal monkey. In A. Elewa (Ed.), Morphometrics for nonmorphometrician Lecture Notes in Earth Sciences (pp. 191-217). Heidelberg, Germany: Springer.

Cardini, A., Jansson, A. U., \& Elton, S. (2007). A geometric morphometric approach to the study of ecogeographical and clinal variation in vervet monkeys. Journal of Biogeography, 34, 1663-1678.

Ceccarelli, D. M., Richards, Z. T., Pratchett, M. S., \& Cvitanovic, C. (2001). Rapid increase in coral cover on an isolated coral reef the Ashmore Reef National Nature Reserve, Northwestern Australia. Marine and Freshwater Research, 62, 1214-1220.

Cowen, R. K., Gawarkiewicz, G., Pineda, J., Thorrold S. R., \& Werner, F. E. (2007). Population connectivity in marine systems an overview. Oceanography, 20(3), 14-21.

Cutress, B. M. (1965). Observations on growth in Eucidaris tribuloides (Lamarck), with special reference to the origin of the oral primary spines. Bulletin of Marine Science, 15, 797-834.

Díaz, P., \& Campos, B. (2014). Ontogenia de la concha larval y postlarval de cuatro especies de bivalvos de la costa del Pacifico sureste. Revista de Biología Marina y Oceanografia, 49(2), 175-191.

Dix, T. G. (1970). Biology of Evechinus chloroticus (Echinoidea: Echinometridae) from different localities. New Zealand Journal of Marine and Freshwater Research, 4, 91-116.

DOF_Diario Oficial de la Federación. (2009). Decreto por el que se declara área natural protegida, con el carácter de Área de Protección de Flora y Fauna, la región conocida como Sistema Arrecifal Lobos-Tuxpan, localizada frente a las costas de los municipios de Tamiahua y Tuxpan, en el Estado de Veracruz. Diario Oficial de la Federación, 659, 79-84.

DOF_Diario Oficial de la Federación. (1992). Decreto por el que se declara área natural protegida, con el carácter de Parque Marino Nacional, la zona conocida como Sistema Arrecifal Veracruzano, ubicada frente a las costas de los municipios de Veracruz, Boca del Río y Alvarado del Estado de Veracruz Llave, con superficie de 52, 238-91-50 hectáreas. Diario Oficial de la Federación, 467, 6-15.
Ebert, T. A. (1968). Growth rates of the sea urchin Strongylocentrotus purpuratus related to food availability and spine abrasion. Ecology, 49, 1075-1091.

Ebert, T. A. (1980). Relative growth of sea urchin jaws: an example of plastic resource allocation. Bulletin of Marine Science, 30, 467-474.

Epherra, L., Crespi-Abril, A., Meretta, P. E., Cledón, M., Morsan, E. M., \& Rubilar, T. (2015). Morphological plasticity in the aristotle's lanter of Arbacia dufresnii (Phymosomatoida: Arbaciidae) off the Patagonian coast. Revista de Biología Tropical, 63(2), 339-351.

Frédérich, B., Adriaens, D., \& Vandewalle, P. (2008). Ontogenetic shape changes in pomacentridae (Teleostei, Perciformes) and their relationships with feeding strategies: a geometric morphometric approach. Biological Journal of the Linnean Society, 97, 92-105.

Frédérich, B., Liu, S. Y. V., \& Dai, C. F. (2012). Morphological and genetic divergences in a coral reef damselfish, Pomacentrus coelestis. Evolutionary Biology, 39, 359-370.

Geissert-Kientz, D. (1999). Regionalización geomorfológica del estado de Veracruz. Investigaciones Geográficas, Instituto de Ecología, 41, 23-47.

Gianguzza, P., \& Bonaviri, C. (2013). Arbacia. En J. M. Lawrence (Ed.), Sea Urchins: Biology and Ecology (pp. 275-283). Florida, United States of America: Elsevier.

González-Gándara, C., Solís-Marín, F. A., De La CruzFrancisco, V., Granados-Barba, A., Salas-Pérez, J. J., Argüelles-Jiménez, J. J., \& Escarcega-Quiroga, P. A. (2015). Riqueza y distribución de equinodermos en los arrecifes del norte y sur de Veracruz, México. Revista de Biología Tropical, 63(2), 183-193.

Hagen, N. T. (2008). Enlarged lantern size in similar-sized, sympatric, sibling species of Strongylocentrotid sea urchins: from phenotypic accommodation to functional adaptation for durophagy. Marine Biology, 153, 907-924.

Hendler, G., Miller, J. E., Pawson, D. L., \& Kier, P. M. (1995). Sea Stars. Sea Urchins, and Allies: Echinoderms of Florida and the Caribbean. Washington, United States of America: Smithsonian Institution Press.

Hendry, A. P., Day, T., \& Taylor, E. B. (2001). Population mixing and the adaptive divergence of quantitative traits in discrete populations: a theoretical framework for empirical tests. Evolution, 55, 459-466.

Hernández, J. C., \& Russell, M. P. (2009). Substratum cavities affect growth-plasticity, allometry, movement and feeding rates in the sea urchin Strongylocentrotus purpuratus. Journal of Experimental Biology, 213, 520-525. 
Jordán-Garza, A. G., González-Gándara, C., Salas-Pérez, J. J., \& Morales-Barragán, A. M. (2017). Coral assemblages are structured along a turbidity gradient on the southwestern Gulf of Mexico, Veracruz. Continental Shelf Research, 138, 32-40.

Kier, P. M. (1975). The Echinoids of Carrie Bow Cay, Belize. Washington, United States of America: Smithsonian Institution Press.

Klingenberg, C. P. (2016). Size, Shape, and form: concepts of allometry in geometric morphometrics. Development Genes and Evolution, 226(3), 113-137.

Krutak, P. R. (1997). Petrography and provenance of siliciclastic sediments, Veracruz-Anton Lizardo reefs, Mexico. En J. San-Joon \& Y. Hi-Il (Eds.), Special Issue. Ocean and Polar Research, 19(3), 231-243.

Lau, D. C. C., Lau, S. C., Qian, P. Y., \& Qiu, J. W. (2009). Morphological plasticity and resource allocation in response to food limitation and hyposalinity in a sea urchin. Journal of Shellfish Research, 28(2), 383-38.

Lawrence, J. M., \& Glynn, P. (1984). Absorption of nutrients from the coral Pocillopora damicornis (L.) by the echinoid Eucidaris thouarsii (val.). Comparative Biochemistry and Physiology, 77(1), 111-112.

Lawrence, J. M., \& Jangoux, M. (2013). Cidaroids. En J. M. Lawrence (Ed.), Sea Urchins: Biology and Ecology (pp. 225-242). Florida, United States of America: Elsevier.

Levitan, D. R. (1988). Density-dependent size regulation and negative growth in the sea urchin Diadema antillarum Philippi. Oecologia, 76, 627-629.

Marcus, N. H. (1980). Genetics of morphological variation in geographically distant populations of the sea urchin, Arbacia punctulata (Lamarck). Journal of Experimental Marine Biology and Ecology, 43, 121-130.

Marcus, N. H. (1983). Phenotypic variability in echinoderms. En M. Jangoux \& J. M. Lawrence (Eds.), Echinoderms studies (pp.19-39). Rotterdam, Netherlands: A. A. Balkema.

Märkel, K. (1979). Strutcture and growth of the Ciradoid socket-join lantern or Aristotle compared to the hinge-join lanterns of non-cidaroid regular echinoids (Echinodermata: Echinoidea). Zoomorphologie, 94, $1-32$.

Márquez, F., \& Van Der Molen, S. (2010). Intraspecific Shell-shape variation in the razor clam Ensis macha along the Patagonia coast. Journal of Molluscan Studies, 77, 123-128.

Márquez, F., Robledo, J., Peñaloza, G. E., \& Van Der Molen, S. (2009). Use of different geometric morphometrics tools for the discrimination of phenotypic stocks of the striped clam Amenghinomya antiqua
(Veneridae) in north Patagonia, Argentina. Fisheries Research, 101, 127-13.

McPherson, B. F. (1968). Contributions to the biology of the sea urchin Eucidaris tribuloides (Lamarck). Bulletin of Marine Science, 18, 400-443.

Mitteroecker, P., \& Bookstein. F. (2011). Linear discrimination, ordination, and the visualization of selection gradients in modern morphometrics. Evolutionary Biology, 38, 100-114.

Nava-Ferrer, M. L., \& Severeyn-Valbuena, H. J. (2011). Variaciones morfológicas en la concha del gasterópodo Pyrgophorus platyrachis como posible respuesta a factores físico-químicos en el Sistema de Maracaibo, Venezuela. Ecotropicos, 24(2), 145-163.

Ortiz-Lozano, L., Pérez-España, H., Granados-Barba, A., González-Gándara, C., Gutiérrez-Velázquez, A., \& Martos, J. (2013). The reef corridor of the Southwest Gulf of Mexico: Challenges for its management and conservation. Ocean \& Coastal Management, 86, 22-32.

Perales-Valdivia, H., Sanay-González, R., \& Marín-Hernández, M. (2015). Thermohaline-structure temporal variation in the Sistema Arrecifal Veracruzano National Park. EBIOS. Special Issue, 2(8), 124-137.

Piñeros, V. J., Ríos-Cárdenas, O, Gutiérrez-Rodríguez, C., \& Mendoza-Cuenca, L. (2015). Morphological differentiation in the damselfish Abudefduf saxatilis along the Mexican Atlantic coast is Associated with Environmental factors and High Connectivity. Evolutionary Biology, 42, 235-249.

Rohlf, F. J. (2016). TpsDig (version 2.26). Stony Brook: Department of Ecology and Evolution, State University of New York at Stony Brook. Retrieved from http://life.bio.sunysb.edu/morph

Salas-Monreal, D., Pérez-Salas, J. J., Salas-de-León, D. A., Monreal-Gómez, M. A., Pérez-España, H., OrtizLozano, L. D., ... Villegas-Sánchez, C. (2017). Corrientes superficiales dentro del Corredor Arrecifal del Suroeste del Golfo de México. UVserva, 3, 32-36.

Salas-Monreal, D., Marín-Hernández, M., Salas-Pérez, J. J., Salas-de-León, D. A., Monreal-Gómez, M. A., \& Pérez-España, H. (2018). Coral reef connectivity within the western Gulf of Mexico. Journal of Marine Systems, 179, 88-99.

Salas-Pérez, J. J., Ocaña-Valencia, A. N., \& GonzálezGándara, C. (2015). Temperatura superficial del mar y concentración de Clorofila-a en zonas arrecifales y desembocaduras de sus ríos en el Golfo de México occidental. En A. Granados-Barba, L. D. OrtizLozano, D. Salas-Monreal \& C. González-Gándara (Eds.), Aportes al conocimiento del Sistema Arrecifal veracruzano hacia el Corredor Arrecifal del Suroeste del Golfo de México (pp. 315-334). Campeche, México: EPOMEX. 
Sánchez, R., Sepúlveda, R. D., Brante, A., \& Cárdenas, L. (2011). Spatial pattern of genetic and morphological diversity in the direct developer Acanthina monodon (Gastropoda: Mollusca). Marine Ecology Progress Series, 434, 121-131.

Santos, S. P., Coutinho, A. B., \& Hajdu, E. (2002). Spongivory by Eucidaris tribuloides from Salvador, Bahia (Echinodermata: Echinoidea). Journal of the Marine Biological Association of the United Kingdom, 83, 295-297.

Schachter-Broide, J., Dujardin, J. P., Kitron, U., \& Gürtler, R. E. (2004). Spatial structuring of Triatoma infestans (Hemiptera, Reduviidae) populations from northwestern Argentina using wing geometric morphometry. Journal of Medical Entomology, 41, 643-649.

Sheets, H. D. (2014a). MakeFan8 (6th version). New York: Department of Physics, Canisius, College, Buffalo. Retrieved from www.canisius.edu/ sheets/ morphsoft.htm

Sheets, H.D. (2014b). PCAGen8 (8th version). New York: Department of Physics, Canisius College, Buffalo. Retrieved from www.canisius.edu/ $\sim$ sheets/morphsoft.html

Sheets, H. D. (2014c). CoordGen8th. New York: Department of Physics, Canisius, College, Buffalo.
Retrieved from www.canisius.edu/ sheets/morphsoft.htm

Slatkin, M. (1987). Gene flow and the geographic structure of natural populations. American Association for the Advancement of Science, 236(4803), 787-792.

Vega-Sánchez, Y. M., Camacho-Morales, E., ChassinNora, O., \& Mendoza-Cuenca, L. (2012). Efecto del tipo de hábitat, genética y selección sexual sobre la morfología alar en Hetarina (Odonata: Calopterygidae). Biológicas, 14, 53-60.

Villegas-Sánchez, C. A., Pérez-España, H., Rivera-Madrid, R., Salas-Monreal, D., \& Arias-González, J. E. (2014). Subtle genetic connectivity between Mexican Caribbean and south-western Gulf of Mexico reefs: the case of bicolor damselfish, Stegastes partitus. Coral Reefs, 33, 241-251.

Zavala-Hidalgo, J., Morey, S. L., \& O’Brien, J. J. (2003). Seasonal circulation on the western shelf of the Gulf of Mexico using a high-resolution numerical model. Journal of Geophysical Research, 108(12), 1-19.

Zelditch, M. L., Swiderski, D. L., \& Sheets, H. (2012). Geometric morphometrics for biologists: a primer ( $2^{\text {nd }}$ ed.). New York, United States of America: Elsevier. 